\title{
DIMENSIONAMENTO DE ENFERMAGEM HOSPITALAR: MODELO OPAS/OMS
}

\author{
Dagmar Willamowius Vituri', Solange Moreira Lima², Cleuza Catsue Takeda Kuwabara², Roseli Broggi Gil", \\ Yolanda Dora Martinez Évora
}

\footnotetext{
${ }^{1}$ Doutoranda em Enfermagem do Programa de Enfermagem Fundamental da Escola de Enfermagem de Ribeirão Preto (EERP) da Universidade de São Paulo (USP). Enfermeira do Hospital Universitário (HU) da Universidade Estadual de Londrina (UEL). Paraná, Brasil. E-mail: dagmar@uel.br

${ }^{2}$ Enfermeira Especialista em Gestão Pública. Assessora Técnica da Diretoria de Enfermagem do HU/UEL. Paraná, Brasil. solange@uel.br

${ }^{3}$ Doutora em Enfermagem. Diretora de Enfermagem do HU/UEL. Paraná, Brasil. cleuzak@yahoo.com.br

${ }^{4}$ Mestranda em Enfermagem do Programa de Enfermagem Fundamental da EERP/USP. Enfermeira do HU/UEL. Paraná, Brasil. auditoriaenf@uel.br

${ }^{5}$ Doutora em Enfermagem. Professora Titular da EERP/USP. São Paulo, Brasil. yolanda@eerp.usp.br
}

\begin{abstract}
RESUMO: O dimensionamento de recursos humanos em enfermagem tem sido ampla e intensamente discutido nos vários âmbitos que envolvem a complexidade do atendimento às necessidades de saúde da população. O objetivo do estudo é dimensionar a enfermagem de um hospital universitário público do Paraná, segundo metodologia proposta pela Rede de Observatórios de Recursos Humanos em Saúde do Brasil. Mediante aplicação da metodologia foi possível dimensionar o quadro de pessoal ideal e comparar com o real, contemplando aspectos de estrutura, processo e especificidades da clientela. Constata-se um déficit de 118 enfermeiros e 204 funcionários de enfermagem de nível médio em relação ao ideal. Espera-se que o presente estudo possa promover discussões entre os enfermeiros do serviço no sentido de aproximá-los do tema e das variáveis que envolvem o dimensionamento de pessoal, pois, atuando diretamente na assistência, possuem condições de avaliar continuamente os recursos disponíveis, subsidiando os estudos desta natureza.

DESCRITORES: Enfermagem. Recursos humanos de enfermagem. Administração de recursos humanos em saúde. Downsizing organizacional.
\end{abstract}

\section{SIZING HOSPITAL NURSING STAFFS: PAHO/WHO MODEL}

\begin{abstract}
Dimensioning nursing human resources has been widely and intensely discussed in the various spheres which involve the complexity of attending the population's health care needs. The objective of this study is to dimension the nursing staff at a Brazilian public university hospital in Paraná according to the methodology proposed by the Brazilian Human Health Resources Observatory Network. In doing so, it has been possible to dimension an ideal staff and compare it with the actual one, contemplating aspects such as structure, process, and client needs. The comparison shows a deficit of 118 nurses and 204 nursing technicians. We hope that the present study may further future discussion among nurses in order to approximate them to the issue and the variables involved in staff-sizing, for if they directly act in providing care, they are in the right position to continuously assess the available resources and subsidize studies of this nature.
\end{abstract}

DESCRIPTORS: Nursing. Nursing staff. Health personnel management. Personnel downsizing.

\section{DIMENSIONES DE LA ENFERMERÍA HOSPITALARIA: MODELO DE LA OPAS/OMS}

RESUMEN: La dimensión de los recursos humanos de enfermería ha sido amplia e intensamente debatida en diversos ámbitos que abarcan la compleja realidad de atender a las necesidades de salud de la población. El objetivo del estudio es establecer las dimensiones de la enfermería de un hospital universitario público del Estado de Paraná, según la metodología propuesta por la Red de Observatorios de Recursos Humanos en Salud del Brasil. La aplicación de la referida metodología permitió obtener la dimensión del equipo de personal ideal y compararlo con el real, al tener en cuenta aspectos, como la estructura, proceso y especificidades de la población atendida. Se constató un déficit de 118 enfermeros y 204 técnicos de enfermería de nivel intermedio. Se espera que con este estudio se pueda fomentar el debate entre los enfermeros del servicio para que se sensibilicen sobre el tema y las variables que intervienen, ya que se encuentran en la posición adecuada para evaluar continuamente los recursos disponibles y colaborar con estudios de esta naturaleza. DESCRIPTORES: Enfermería. Personal de enfermería. Administración del personal en salud. Reducción de personal. 


\section{INTRODUÇÃO}

A questão do dimensionamento de recursos humanos em enfermagem tem permeado as inúmeras esferas da complexidade do atendimento, dentre elas a qualidade do cuidado, resultados da atenção, satisfação do cliente, carga de trabalho, horas de assistência de enfermagem, assim como contenção de custos, situação que se faz concreta em grande parte das instituições de saúde do Brasil e do mundo. ${ }^{1}$

As transformações socioculturais, políticas e econômicas, ao incidirem sobre as políticas organizacionais, afetam diretamente a enfermagem sob a perspectiva da redução de custos, ${ }^{2-3}$ contribuindo para a manutenção da lógica capitalista de aumento da produtividade, muitas vezes em detrimento do cuidado de qualidade, em razão de que a enfermagem representa o maior contingente de trabalhadores das instituições de saúde. ${ }^{4-5}$

A determinação do número e da composição da equipe se dá, dentre outros critérios, pelo tipo e complexidade do serviço prestado. Para tanto, uma análise da organização do trabalho deve considerar os diferentes processo e as diferenças institucionais, além das necessidades de atenção à saúde e o modelo assistencial adotado. ${ }^{4}$

Surgem assim as discussões sobre "carga de trabalho em enfermagem" e a sua relação com a qualidade da assistência e a segurança do paciente. São vários os fatores que contribuem para um atendimento livre de riscos, dos quais se destaca a alocação de recursos humanos nas unidades hospitalares. ${ }^{6}$

O dimensionamento de recursos humanos é uma atividade/habilidade gerencial do enfermeiro, que envolve a previsão de pessoal sob os enfoques quantitativo e qualitativo, com vista ao atendimento das necessidades da clientela, na busca de uma melhor qualidade possível da atenção. ${ }^{7-8}$ Mediante uma previsão apropriada de pessoal de enfermagem, as instituições de saúde podem racionalizar custos e otimizar a dinâmica assistencial. ${ }^{9}$

No transcorrer da construção do conhecimento da profissão, vários estudos foram desenvolvidos no sentido de desenvolver e propor metodologias de cálculo de pessoal em enfermagem. Dentre eles, tem-se a Resolução do Conselho Federal de Enfermagem (COFEN) n ${ }^{0}$ 293/2004 ${ }^{10}$, a qual determina que, para o dimensionamento de pessoal, devem ser consideradas as características da instituição e do serviço de enfermagem, assim como a fundamentação legal do exercício profissional Lei $n^{0} 7.498 / 86^{11}$ e o Código de Ética dos Profissionais de Enfermagem ${ }^{12}$, além de outras Re- soluções e Decisões do Sistema COFEN/COREN. Para implementação da metodologia de cálculos, a clientela atendida deve ser qualificada segundo um Sistema de Classificação de Pacientes (SPC). ${ }^{10}$

Estudos ${ }^{7,13}$ que discorrem sobre dimensionamento de pessoal de enfermagem fazem referência à tese de Livre Docência ${ }^{14}$, em que a autora apresenta a evolução histórica da temática, relatando que, antes de 1939, utilizava-se o método intuitivo ou das relações; em 1939, introduziu-se a variável horas-médias de cuidado; em 1947, as variáveis relativas a ausências e, em 1960, o SPC.

No Brasil, foram desenvolvidas várias metodologias de SPC que consideram a dependência dos pacientes em relação ao cuidado de enfermagem, ${ }^{15-17}$ assim como, existem outras metodologias de classificação de pacientes, fundamentadas na concepção de carga de trabalho, como o Therapeutic Intervention Scoring Sistem (TISS) que se utiliza da variável gravidade como diretamente relacionada ao número de intervenções terapêuticas e horas necessárias de assistência. ${ }^{7}$

Pesquisas têm sido desenvolvidas no sentido de propor novas metodologias de dimensionamento de pessoal, dentre as quais se destaca o estudo desenvolvido pela Estação Observatório de Recursos Humanos de São Paulo, que compõe a Rede Observatório de Recursos Humanos em Saúde do Brasil, que é uma rede de cooperação técnica regulamentada pelo Ministério da Saúde e apoiada pela Organização Pan-Americana da Saúde (OPAS)/Organização Mundial da Saúde (OMS). O fruto desse trabalho em rede foi uma proposta de dimensionamento de pessoal para hospitais gerais públicos, no contexto do Sistema Único de Saúde (SUS) de São Paulo, sendo, porém, aplicável às várias realidades deste nosso imenso país. A metodologia propõe a utilização do parâmetro "hora assistencial por paciente" e preconiza que os responsáveis pelo gerenciamento de recursos humanos das instituições considerem, além das diretrizes apresentadas na proposta, as peculiaridades institucionais, orientando-se pelos eixos estratégicos. ${ }^{18}$

Para a OPAS/OMS no Brasil, redes podem ser consideradas mecanismos que permitem o compartilhamento de informações, experiências e a valorização do processo de comunicação. Ao incorporar este conceito, a OPAS/OMS visa garantir a visibilidade e a confiabilidade da Organização, por meio da socialização de informações sobre as ações e seus resultados. ${ }^{19}$

No contexto da prática de gerenciamento de recursos humanos de enfermagem na instituição 
em estudo, adotou-se, até o ano de 2007, um método ${ }^{20}$ que utiliza variáveis de estrutura e processo de trabalho, aliadas à variável Índice de Segurança Técnica (IST), contudo não considera as características de dependência/necessidades dos pacientes atendidos, mas sim a variável "postos de trabalho".

Sentiu-se, porém, a necessidade de buscar novas propostas de cálculo de pessoal, motivada pela mudança no perfil dos pacientes atendidos na instituição e pela constante permanência de pacientes graves e dependentes nas unidades de internação médico cirúrgica, fato este decorrente do número insuficiente de leitos de terapia intensiva no município, para atender à demanda da $17^{\mathrm{a}}$ Regional de Saúde do Paraná, à qual está vinculado o hospital.

A metodologia proposta pela Resolução COFEN 293/2004 ${ }^{10}$, assim como outras que preconizam o dimensionamento de pessoal em enfermagem segundo o SCP, prevê um incremento expressivo no quantitativo de pessoal de enfermagem da instituição, tendo como foco a melhoria da qualidade da assistência. Contudo, esse déficit apresentado, aliado à necessidade de suprir um IST elevado devido às características institucionais, acabava por dificultar o gerenciamento dos conflitos internos relativos à carência de pessoal, somando-se à falta de governabilidade da Direção sobre a provisão de recursos humanos, realidade das instituições públicas do país.

Diante deste contexto e da necessidade de se rever o grau de dependência da assistência de enfermagem adotado na instituição, sem perder o foco na qualidade da atenção, foi adotada a metodologia de dimensionamento proposta pela Rede de Observatórios de Recursos Humanos em Saúde do Brasil. Para tanto, foi elaborado um rol de critérios de avaliação para implementação da proposta, segundo as características e realidade locais, aliadas à vivência da prática de enfermagem na instituição. Foram organizados os critérios para determinação do IST e dados a respeito das maiores taxas de ocupação das unidades de assistência nos últimos cinco anos (2005 a 2009), as médias de pacientes por especialidades em cada uma das unidades e as maiores médias de atendimento nas demais unidades de apoio diagnóstico e tratamento do hospital.

Sendo assim, o presente estudo teve como objetivo comparar o quadro de recursos humanos de enfermagem de um hospital universitário público do estado do Paraná com o quantitativo ideal, segundo a metodologia proposta pela Rede de Observatórios de Recursos Humanos em Saúde do Brasil.

\section{MÉTODO}

Trata-se de um estudo descritivo, autorizado pela diretoria da instituição, que aborda a implementação da metodologia de dimensionamento de pessoal em enfermagem proposta pela Rede de Observatórios de Recursos Humanos em Saúde do Brasil. ${ }^{18}$

Os dados foram coletados a partir do banco de dados de recursos humanos da Diretoria de Enfermagem, referentes ao mês de maio de 2010. O presente estudo é parte de um projeto aprovado pelo Comitê de Ética da instituição, sob o Parecer de ${ }^{\mathrm{o}}$ 108/06 CAAE 0083.0.268.000-06.

O hospital em estudo possui 317 leitos destinados, exclusivamente, para atendimento ao Sistema Único em Saúde (SUS), nas mais diversas especialidades. É referência em trauma e gestação de alto risco. Trata-se de um hospital universitário, com estrutura pavilhonar da década de 1970, com enfermarias de 3 a 6 leitos, distribuídos em unidades assistenciais de até 64 leitos e que desenvolve, além da prática assistencial, o ensino, a pesquisa e a extensão à comunidade.

A Diretoria de Enfermagem é composta por um quadro de 732 servidores, dos quais 122 são contratados como enfermeiros, 186 como técnicos em enfermagem e 424 como auxiliares de enfermagem. O processo de trabalho de enfermagem se dá na lógica do cuidado integral, em que o indivíduo é assistido em suas necessidades de enfermagem pelo mesmo funcionário.

Os procedimentos adotados para o desenvolvimento do estudo estão descritos em fases, com o intuito de tornar a apresentação mais didática e facilitar a compreensão.

Na primeira fase, buscou-se a compreensão da lógica proposta pelo método e observou-se que a metodologia considera algumas variáveis também nas demais propostas já existentes sobre dimensionamento de pessoal em enfermagem, como por exemplo: IST, número de leitos, taxa de ocupação, carga de trabalho, jornada de trabalho, número de dias trabalhados, produtividade, dentre outras.

Na segunda fase, foi realizado um levantamento da produtividade de todas as unidades de atendimento, apoio diagnóstico e tratamento, assim como das taxas de ocupação das unidades de internamento referentes aos últimos cinco anos (2005 a 2009). Foram adotados como parâmetro de cálculo os maiores valores encontrados.

$\mathrm{Na}$ terceira fase, foi determinado o percentual de pacientes por especialidade, conforme a 
realidade das internações na instituição, de forma que a presença de pacientes dependentes e em risco de morte fosse contemplada no perfil de cada unidade. Para tanto, além das especialidades comuns a cada área como, por exemplo, clínica médica e clínica cirúrgica, foi estimada uma média de pacientes com necessidades de cuidados semi-intensivos e intensivos, de acordo com a realidade histórica de cada uma das unidades assistenciais.

$\mathrm{Na}$ quarta fase determinou-se o IST da equipe de enfermagem, considerando para tal as ausências previstas e não previstas em relação à carga horária contratada, bem como um percentual de horas destinadas às atividades de educação permanente. Obteve-se como resultado um Coeficiente de IST, que resulta da seguinte equação:

$$
\text { IST }=\frac{\text { Horas contratadas por semana }}{\text { Horas reais trabalhadas por semana }}
$$

Horas reais trabalhadas se refere à jornada efetivamente cumprida pelo empregado, durante uma semana, excluídas todas as ausências ao serviço, como faltas, folgas, feriados, finais de semana, férias, licença médica, licença maternidade e outras, como a participação em cursos, congressos e eventos que envolvem atividades educativas. ${ }^{18}$

Para determinação do coeficiente de IST da Diretoria de Enfermagem do hospital em estudo, estimaram-se as ausências previstas e não previstas apresentadas no quadro 1.

Quadro 1 - Ausências previstas e não previstas consideradas para determinação do IST da Diretoria de Enfermagem. Londrina-PR, 2010

\begin{tabular}{|l|c|}
\hline Itens & Dias úteis \\
\hline Ausências previstas & 22 \\
\hline Férias & 52 \\
\hline Domingos & 16 \\
\hline Feriados/recessos & 13 \\
\hline $\begin{array}{l}\text { Licença especial (recesso final } \\
\text { de ano) }\end{array}$ & 18 \\
\hline Licença prêmio & 30 \\
\hline Ausências não previstas & 11 \\
\hline Atestados e licenças médicas & 162 \\
\hline Educação permanente & $\mathbf{3 6}$ horas semanais \\
\hline Total de dias úteis & $\mathbf{2 4}$ horas semanais \\
\hline Horas contratuais & \\
\hline $\begin{array}{l}\text { Horas contratuais em } \\
\text { unidades com periculosidade }\end{array}$ & \\
\hline
\end{tabular}

Cabe ressaltar que, para o critério "atestados e licenças médicas", foi considerado que o servidor público da instituição em estudo possui o "direito" de apresentar três atestados médicos/ mês e que, dos doze meses do ano, o servidor estará ausente por motivo de férias, recesso de final de ano e licença prêmio num quantitativo de dias equivalente a dois meses, ou seja, dos doze meses do ano trabalhará dez meses. Desse modo, dez meses multiplicados por três atestados de um dia/mês equivalem a trinta dias de ausências não previstas.

Com relação à Licença Prêmio, ao servidor da instituição, a cada cinco anos trabalhados, é concedido, segundo o Plano de Carreira, Cargos e Salários, o direito de usufruir três meses de licença remunerada. Para o cálculo do impacto desse tipo de ausência, houve a divisão de 90 dias por cinco anos, resultando no número de 18 dias de ausência/ano.

Para o desenvolvimento deste estudo, não se considerou a ausência por licença maternidade, em razão de problemas informacionais que não permitem conhecer a taxa de natalidade entre a população de servidores da instituição.

A partir da aplicação das varáveis relacionadas, chegou-se a um coeficiente de IST de 1,46, ou seja, ao quadro de pessoal se faz necessário um incremento de servidores de $46 \%$ em razão da necessidade de cobertura das ausências previstas e não previstas, que representam um percentual bastante elevado quando comparado à realidade das instituições privadas.

$\mathrm{Na}$ quinta fase, os cálculos propostos pela metodologia foram aplicados para todas as unidades de assistência, considerando-se o quantitativo de horas assistenciais proposto por Sala, ${ }^{18}$ que é determinado segundo a especialidade/clínica dos pacientes: Cirurgia Ambulatorial - 3 horas; Clínica Médica, Berçário Normal/Alojamento Conjunto - 4 horas; Recuperação Anestésica e Observação de Pronto Socorro - 5 horas; Clínica Cirúrgica, Ginecológica e Psiquiátrica - 5,5 horas; Clínica Pediátrica, Obstétrica e Pré-Parto - 6 horas; Clínica MI/AIDS - 7 horas; Semi Intensivo - 8,5 horas; Clínica de Queimados - 10 horas e UTI Geral e Neonatal - 12 horas.

A partir da coleta e organização das variáveis, procedeu-se à aplicação do seguinte cálculo para dimensionamento de pessoal de enfermagem em unidades de internamento: 
$\begin{gathered}\mathrm{N}^{\circ} \text { de pessoal de } \\ \text { enfermagem }\end{gathered}=\frac{\mathrm{N}^{\circ} \text { de leitos } X \text { Taxa de ocupação } X \mathrm{~N}^{\circ} \text { de horas assistenciais } X \text { dias da semana } X \text { IST }}{\text { Jornada semanal }}$

Mediante o resultado do cálculo, a metodologia determina que do total de funcionários necessários se destine, de forma geral, $20 \%$ para o quadro de enfermeiros e $80 \%$ para o quadro de pessoal de enfermagem de nível médio, com ressalva para unidades especializadas. ${ }^{18}$

Para o cálculo foram considerados os leitos cadastrados na instituição, bem como a realidade da presença de pacientes que necessitam de cui- dados semi-intensivos e intensivos nas unidades de internamento. Dessa forma, do quantitativo de leitos cadastrados por unidade, foi deduzido um número médio de leitos, normalmente ocupados por pacientes internados com necessidade de cuidados semi-intensivos e intensivos, para os quais foram computadas horas assistenciais específicas dessas especialidades. $\mathrm{O}$ quadro 2 apresenta a situação descrita.

Quadro 2 - Quantitativo de leitos cadastrados no SUS e o número médio de leitos frequentemente ocupados por pacientes que necessitam de cuidados semi-intensivos e intensivos (leitos informais). Londrina-PR, 2010

\begin{tabular}{|c|c|c|c|c|}
\hline \multirow{2}{*}{ Unidades } & \multicolumn{2}{|c|}{ Leitos cadastrados } & \multicolumn{2}{|c|}{ Leitos informais } \\
\hline & Internação & UTI & UTI & Semi \\
\hline Centro de tratamento de queimados & 10 & 06 & - & - \\
\hline Unidade feminina & 47 & - & 03 & 06 \\
\hline Unidade masculina & 74 & - & 03 & 07 \\
\hline Unidade de moléstias infecciosas & 30 & - & 03 & - \\
\hline Unidade de tisiologia & 10 & - & 01 & - \\
\hline Unidade de pronto socorro & 48 & - & 07 & - \\
\hline Unidade de maternidade & 19 & - & - & 03 \\
\hline Unidade de pediatria & 34 & - & - & 06 \\
\hline Unidade de UCI neonatal & 10 & - & 05 & - \\
\hline Unidade de UTI neonatal & - & 07 & - & - \\
\hline Unidade de UTI pediátrica & - & 05 & - & - \\
\hline Unidade de UTI adulto 1 & - & 10 & - & - \\
\hline Unidade de UTI adulto 2 & - & 07 & - & - \\
\hline Total & 282 & 35 & 22 & 22 \\
\hline
\end{tabular}

Para as unidades de atendimento, apoio diagnóstico e tratamento, a metodologia prevê a aplicação da variável produtividade, conforme os cálculos a seguir:

$$
\begin{gathered}
\mathrm{N}^{\circ} \text { de enfermeiros }=\frac{\mathrm{N}^{\circ} \text { de procedimentos/atendimentos/paciente-dia/salas funcionando X } \mathrm{N}^{\circ} \text { de horas }}{\text { assistenciais } \mathrm{X} \text { dias da semana X IST }} \\
\begin{array}{c}
\text { Jornada semanal } \\
\mathrm{N}^{\circ} \text { de Auxiliares/Técni- } \\
\text { cos de enfermagem }
\end{array}= \\
\mathrm{N}^{\circ} \text { de procedimentos/atendimentos/paciente-dia/salas funcionando X } \mathrm{N}^{\circ} \text { de hor } \\
\text { assistenciais X dias da semana X IST }
\end{gathered}
$$

Para essas unidades foram considerados parâmetros de cálculo específicos, que consideram aspectos de estrutura, produtividade e horas assistenciais por especialidade. Cabe ressaltar que, para essas unidades, a metodologia prevê um quantitativo de horas assistenciais diferenciado para enfermeiros e pessoal de enfermagem de nível médio, de acordo com o tempo médio de atenção dispensado pela categoria profissional. ${ }^{18}$ 


\section{RESULTADOS E DISCUSSÃO}

Mediante aplicação dessa metodologia foi possível dimensionar o quadro de pessoal de enfermagem ideal e estabelecer uma comparação com o quadro real.

Porém, o ideal nem sempre é exequível, principalmente quando se trata de instituições públicas, onde os enfermeiros não possuem governabilidade sobre a contratação de pessoal. Além disso, a contenção de despesas imposta a todos os ramos da sociedade organizada interfere sobre a contratação destes profissionais. ${ }^{4-5}$

Contudo, cabe ressaltar que a lógica capitalista visa à produtividade a um menor custo, porém produzir na área da saúde não equivale ao modo industrial de produzir, pois o serviço de saúde não se concretiza em coisas materiais, assim como o custo não diminui, pelo contrário, a tendência é elevar continua e progressivamente à medida da incorporação de novas tecnologias. ${ }^{4-5,21}$

A tabela 1 apresenta o quantitativo de pessoal de enfermagem de nível superior e nível médio atual, por unidade assistencial e estabelece um comparativo com o ideal, segundo a metodologia estudada.

No cômputo geral é possível observar um déficit instalado de 118 profissionais enfermeiros e 204 profissionais de enfermagem de nível médio.

Tabela 1 - Comparativo entre o número de funcionários da Diretoria de Enfermagem no ano de 2010 em relação ao número ideal, segundo a metodologia estudada. Londrina-PR, 2010

\begin{tabular}{|c|c|c|c|c|}
\hline \multirow{3}{*}{ Unidades } & \multicolumn{4}{|c|}{$N^{0}$ de funcionários } \\
\hline & \multicolumn{2}{|c|}{ Atual } & \multicolumn{2}{|c|}{ Ideal } \\
\hline & Enfermeiro & Aux./Tec. & $\begin{array}{c}\text { Enfermeiro } \\
20 \%\end{array}$ & $\begin{array}{c}\text { Aux./Tec. } \\
80 \%\end{array}$ \\
\hline Centro de tratamento de queimados & 10 & 40 & 13 & 47 \\
\hline Unidade feminina & 5 & 45 & 14 & 57 \\
\hline Unidade masculina & 11 & 57 & 20 & 82 \\
\hline Unidade de moléstias infecciosas & 4 & 20 & 8 & 33 \\
\hline Unidade de tisiologia* & - & 3 & 5 & 12 \\
\hline Unidade de pronto socorro & 13 & 91 & 30 & 121 \\
\hline Unidade de maternidade & 4 & 22 & 7 & 27 \\
\hline Unidade de pediatria† & 6 & 32 & 9 & 34 \\
\hline Unidade de UCI neonatal & 2 & 18 & 8 & 30 \\
\hline Unidade de UTI neonatal & 4 & 18 & 6 & 23 \\
\hline Unidade de UTI pediátrica ${ }^{\dagger}$ & 2 & 16 & 4 & 12 \\
\hline Banco de leite humano & 1 & 3 & 1 & 5 \\
\hline Unidade de UTI adulto & 6 & 31 & 7 & 27 \\
\hline Unidade de UTI adulto* & 4 & 23 & 5 & 19 \\
\hline Centro cirúrgico & 6 & 42 & 8 & 63 \\
\hline Centro de esterilização de material & 2 & 32 & 5 & 42 \\
\hline Ambulatório do $\mathrm{HU}$ & 1 & 3 & 15 & 12 \\
\hline Eletrodiagnóstico ${ }^{\ddagger}$ & 1 & 5 & 2 & 12 \\
\hline Endoscopia $\ddagger$ & 1 & 3 & 2 & 7 \\
\hline Hemocentro & 1 & 17 & 7 & 8 \\
\hline Hemodinâmica & 3 & 8 & 10 & 40 \\
\hline Quimioterapia & 1 & 2 & 1 & 5 \\
\hline Radiologia ${ }^{\ddagger}$ & 1 & 10 & 2 & 22 \\
\hline Tratamento dialítico & 1 & 10 & 3 & 29 \\
\hline Ambulatório do hospital de clínicas & 7 & 34 & 23 & 20 \\
\hline Total & 97 & 585 & 215 & 789 \\
\hline
\end{tabular}

* O resultado inicial do cálculo apresentava o número de quatro enfermeiros como mínimo necessário, já incluso o IST, porém se considerou a necessidade de pelo menos um enfermeiro por turno de trabalho e, a partir disso, aplicou-se o coeficiente de IST. Como resultado obteve-se o número de cinco enfermeiros.

† As unidades de Pediatria e UTI Pediátrica estão situadas na mesma estrutura física, portanto, foi considerado um IST para as duas unidades em conjunto.

‡ Para as unidades de Eletrodiagnóstico, Endoscopia e Radiologia foi considerada a necessidade de um enfermeiro por turno de funcionamento, em esquema de supervisão direta e semi-direta, acrescido de IST, totalizando seis enfermeiros, os quais foram distribuídos nas três unidades. 
Quanto ao enfermeiro, o maior déficit em relação ao ideal está nas unidades médico-cirúrgicas Feminina, Masculina, Moléstias Infecciosas, Tisiologia, Pronto Socorro e UCI Neonatal. Nas unidades de Apoio Diagnóstico e Tratamento, o déficit se destaca no Ambulatório do HU e do Hospital de Clínicas, Hemocentro e Hemodinâmica.

No que se refere aos profissionais de nível médio, os maiores déficits estão na Unidade Masculina, Tisiologia, Pronto Socorro, Centro Cirúrgico, Ambulatório do HU, Eletrodiagnóstico, Hemodinâmica, Radiologia e Tratamento Dialítico.

As unidades UTI Pediátrica, UTI Adulto 1 e UTI Adulto 2 apresentaram um número maior de funcionários de nível médio do que o recomendado como ideal. Porém se faz necessário analisar em paralelo o quadro de enfermeiros destas unidades, o qual se encontra abaixo do recomendado. Essa análise se faz importante, pois a tendência é que essas duas categorias estejam numa relação de compensação, mesmo contrariando o estabelecido na Lei ${ }^{\circ}$ 7.498/86, que regulamenta o exercício da profissão. ${ }^{11}$

A estrutura física compartimentada e o processo de trabalho fragmentado, característicos das unidades de Hemocentro e Ambulatório do Hospital de Clínicas justificam saldo positivo de servidores de nível médio apresentado.

A partir da avaliação numérica do quadro de pessoal de enfermagem da instituição, foi avaliado o perfil desses servidores, no sentido de agregar à constatação do déficit quantitativo uma estimativa da situação funcional destes, usando como referência o ano de $2009 .{ }^{22}$ A variável situação funcional considera a condição de dupla jornada de trabalho e readaptação funcional, que diz respeito aos funcionários que se encontram impedidos, temporária ou definitivamente, por motivo de doença, de exercer as funções do cargo para o qual foram nomeados. Foram também estimadas as possíveis aposentadorias até o ano de 2014 e denominou-se, no presente estudo, o servidor que se encontra nessa situação de "pré-aposentado".

A partir dos dados da tabela 2, é possível constatar que o maior percentual $(67 \%)$ de servidores lotados na Diretoria de Enfermagem do hospital em estudo encontra-se na faixa entre 40 e 59 anos, caracterizando um percentual em enve- lhecimento segundo a OMS, que adota o conceito de "trabalhador em envelhecimento" para o indivíduo a partir de 45 anos de idade, período em que a capacidade funcional necessária ao desempenho de certas tarefas começa a declinar. ${ }^{23-24}$

Tabela 2 - Perfil dos servidores da Diretoria de Enfermagem, segundo as faixas etárias. Londrina-PR, 2010

\begin{tabular}{lc}
\hline Faixa etária & Percentual \\
\hline 20 a 39 anos & $27 \%$ \\
40 a 59 anos & $67 \%$ \\
Mais que 60 anos & $6 \%$ \\
\hline Total & $\mathbf{1 0 0 \%}$ \\
\hline
\end{tabular}

Fonte: Assessoria Técnica da Diretoria de Enfermagem, 2010.

No cotidiano da prática de enfermagem se observa que quanto maior o período de atuação profissional na área, maiores os riscos de doenças profissionais ou doenças do trabalho decorrentes de atividades ditas "pesadas" e insalubres. Isto pode ser considerado pelos altos índices de absenteísmo não previsto na instituição, que, na média, situa-se em $6,8 \%$, porém em determinados períodos e unidades de assistência, este percentual chegou a $26 \%$ do quadro de pessoal afastado por motivos relacionados à saúde física e emocional. ${ }^{22}$ Essa relação também pode ser inferida a partir da constatação do quantitativo de servidores readaptados nas unidades de UTI Adulto 1 e Ambulatório do Hospital de Clínicas, que apresentam respectivamente quatro e seis funcionários impedidos de exercerem as funções do cargo.

Os dados apresentados na tabela 3 demonstram um representativo percentual de servidores de enfermagem que possuem outro emprego ou estudam em concomitância à atuação na instituição. Novamente vem à tona a questão da sobrecarga de trabalho, comprometimento da produtividade e da saúde física e emocional e, na prática diária, percebe-se que o maior número de ausências não previstas é apresentado por servidores que possuem outras atividades funcionais e/ ou acadêmicas em concomitância, e/ ou encontram-se em situação de readaptação funcional. Contudo, não foi objeto deste estudo desvendar o nível de relação entre estas variáveis, o que existe a necessidade de estudos mais aprofundados.

Tabela 3 - Perfil dos servidores da Diretoria de Enfermagem, segundo a situação funcional. LondrinaPR, 2010

\begin{tabular}{lcccc}
\hline Situação funcional & Dupla jornada trabalho & Dupla jornada estudo & Readaptados & Pré-aposentados \\
\hline Percentual & $14,5 \%$ & $17,5 \%$ & $5 \%$ & $10,3 \%$ \\
\hline Fonte: Assessoria Técnica da Diretoria de Enfermagem, 2010. & & & \\
\hline
\end{tabular}


Essa relação foi contextualizada em estudo desenvolvido em um hospital universitário do oeste do Paraná. ${ }^{25}$ As autoras encontraram um percentual significativo de ausências não previstas ao trabalho por motivos de saúde e refletiram se não seria um indicativo de sobrecarga de trabalho. No referido estudo, a permanência de pacientes com necessidades de cuidados semi-intensivos e intensivos em unidades médico cirúrgicas também era uma prática.

Resultado diferente foi encontrado em estudo desenvolvido em um hospital do Estado de São Paulo. ${ }^{13}$ As autoras não encontraram associação entre licenças médicas e os locais de trabalho, pois o maior número de licenças era de servidores que não atuavam em unidades de terapia intensiva que, segundo elas, seriam mais estressantes e com maior risco ocupacional.

Com base nos resultados encontrados, fica evidente o déficit significativo no quadro de pessoal da Diretoria de Enfermagem da instituição, bem como certas dificuldades no gerenciamento do quadro existente. Resultado semelhante foi encontrado em outras pesquisas, ${ }^{9 ; 25}$ porém realidades em que se constatou um quantitativo de funcionários acima do que os cálculos apresentavam como condição ideal foram encontradas, o que caracteriza uma heterogeneidade na forma de gerenciar recursos humanos de enfermagem no país. ${ }^{3,6}$ Salienta-se que os estudos acima utilizaram metodologias distintas de dimensionamento de pessoal de enfermagem, não cabendo o estabelecimento de correlações entre eles.

\section{CONSIDERAÇÕES FINAIS}

O estudo da metodologia de dimensionamento de pessoal, desenvolvida por especialistas na temática, que envolve o gerenciamento de recursos humanos em saúde, fruto da filosofia do trabalho em redes, estimulada e propagada pela OPAS, permitiu adaptar a proposta às variáveis da instituição e implementá-la como ferramenta gerencial efetiva e eficaz.

O desenvolvimento do presente estudo possibilitou o dimensionamento de pessoal de enfermagem na instituição, considerando aspectos de estrutura, processo e necessidades da clientela. Por meio desta proposta foi possível considerar as especificidades das unidades da instituição estudada e as necessidades de assistência dos pacientes atendidos, com base num levantamento histórico das variáveis de estudo.
Ressalta-se que o estudo não se propôs avaliar se o quantitativo de pessoal determinado pela aplicação desta metodologia garantirá uma assistência de qualidade melhor, mesmo porque as variáveis que influenciam o resultado da atenção não se restringem ao dimensionamento de pessoal, embora este seja um importante indicador.

Com base nos resultados encontrados, constata-se um déficit significativo no quadro de pessoal da Diretoria de Enfermagem do hospital. Em nível gerencial local, esses dados são utilizados como ferramenta para o gerenciamento dos recursos humanos da Diretoria, subsidiando a redistribuição/remanejamento interno, com vista a prover melhorias nas unidades médico cirúrgicas para o atendimento das necessidades dos pacientes que demandam de cuidados semi- intensivos e intensivos.

Considerando a falta de governabilidade do enfermeiro, principalmente em instituições públicas, com relação à reposição/ampliação do quadro de recursos humanos de enfermagem, acredita-se que os dados obtidos através do adequado dimensionamento de pessoal possam subsidiar e fundamentar discussões com os Órgãos competentes, no sentido de prover pessoal suficiente na busca de uma melhor qualidade da atenção, independentemente da metodologia escolhida, desde que reflita as reais necessidades locais.

Espera-se que o presente estudo alerte os enfermeiros para a necessidade de buscar formas e metodologias que fundamentem as reivindicações de pessoal junto aos Órgãos competentes e subsidiem a previsão de recursos humanos para projetos de ampliação e criação de unidades de assistência, assim como possa promover discussões entre os enfermeiros do serviço estudado no sentido de aproximá-los do tema e das variáveis que envolvem a questão, pois este é o profissional que atua diretamente na assistência, estando mais apto para realizar o dimensionamento de pessoal de enfermagem, em razão de possuir condições de avaliar, continuamente, os recursos disponíveis e as necessidades dos pacientes.

O uso sistemático de informações geradas a partir da aplicação de uma metodologia cientificamente fundamentada para o dimensionamento de pessoal é imprescindível, porém se reitera a reflexão de que o dimensionamento de recursos humanos como um indicador não será percebido como tal enquanto não for utilizado para relacionar quantidade de servidores com qualidade da assistência. Destaca-se este ponto como limitação 
do presente estudo e recomenda-se o desenvolvimento de pesquisas no sentido de estabelecer essa relação.

\section{REFERÊNCIAS}

1. Madalosso MARM, Patrício ZM. Refletindo sobre a qualidade do cuidado de enfermagem: uma proposta assistencial transformadora. Texto Contexto Enferm. 2000 Mai-Ago; 9(2 pt.2):562-76.

2. Rogenski KE, Fugulin FMT, Gaidzinski RR, Rogenski NMB. Tempo de assistência de enfermagem em instituição hospitalar de ensino. Rev Esc Enferm. USP [online]. 2011 Mar [acesso 2011 Abr 07]; 45(1): Disponível em: http://www.scielo.br/scielo. php?pid $=$ S0080-62342011000100031\&script $=$ sci arttext.

3. Antunes AV, Costa MN. Dimensionamento de pessoal de enfermagem em um hospital universitário. Rev Latino-am Enferm. 2003 NovDez; 11(6):832-9.

4. Matos E, Pires D. Teorias administrativas e organização do trabalho: de Taylor aos dias atuais, influências no setor saúde e na enfermagem. Texto Contexto Enferm [online]. 2006 Set [acesso 2011 Abr 07]; 15(3): Disponível em: http:/ / www.scielo.br/ scielo.php?pid=S010407072006000300017\&script $=$ sci_arttext.

5. Santos Filha AC, Silva CJF, Teixeira CAC, Soares CA, Dorfey ES, Oliveira GN, et al. Dimensionamento de pessoal em enfermagem. Revista Digital de Pesquisa CONQUER [online]. 2008[acesso 2011 Abr 07]; 3. Disponível em: http://www.fasb.edu.br/revista/ index.php/conquer/article/view/86/63.

6. Krokoscz DVC. Efeitos da alocação de pessoal e da carga de trabalho de enfermagem nos resultados da assistência em unidade de internação médico cirúrgica [dissertação]. São Paulo (SP): Universidade de São Paulo, Escola de Enfermagem; 2007.

7. Campos LF, Melo MRACM. Dimensionamento de pessoal de enfermagem nos hospitais de Ribeirão Preto-SP. Cogitare Enferm. 2007 AbrJun;14(2):237-46.

8. Gaidzinski RR, Fugulin FMT, Castilho V. Dimensionamento de pessoal de enfermagem em instituições de saúde. In: Kurcgant P, coordenador. Gerenciamento de enfermagem. São Paulo (SP): Guanabara Koogan; 2005. p.125-37.

9. Inoue KC, Matsuda LM. Dimensionamento da equipe de enfermagem da UTI-adulto de um hospital ensino. Rev Eletr Enf [online]. 2009 [acesso 2010 Mar. 23]; 11(1): Disponível em: http:/ /www. fen.ufg.br/revista/v11/n1/v11n1a07.htm.

10. Conselho Federal de Enfermagem. Resolução COFEN no 293, de 21 de setembro de 2004. Fixa e estabelece parâmetros para dimensionamento do quadro de profissionais de enfermagem nas unidades assistenciais das instituições de saúde e assemelhados. [online]. [acesso 2010 Mar 23]. Disponível em: http://www.portalcoren-rs.gov. br/web/resoluca/r293.htm.

11. Conselho Federal de Enfermagem. Lei n. 7.498/86, de 25 de junho de 1986. Dispõe sobre a regulamentação do exercício da enfermagem e dá outras providências [online]. [acesso 2010 Mar 10]. Disponível em: http://www.portalcofen.gov.br/2007/materias. asp? ArticleID $=22 \&$ sectionID $=35$.

12. Conselho Federal de Enfermagem. Resolução COFEN n ${ }^{\circ} 311 / 2007$. Aprova a reformulação do código de ética dos profissionais de enfermagem [online]. [acesso 2010 Mar 03]. Disponível em: http://www.portalcofen.gov.br/2007/materias. asp? ArticleID $=7221 \&$ sectionID $=34$.

13. Matsushita MS, Adami NP, Carmagnani MIS. Dimensionamento do pessoal de enfermagem das unidades de internação do Hospital São Paulo. Acta Paul Enferm. 2005 Mar; 18(1):9-19.

14. Gaidzinski RR. Dimensionamento de pessoal de enfermagem em instituições hospitalares [tese]. São Paulo (SP): Universidade de São Paulo, Escola de Enfermagem; 1998.

15. Perroca MG. Sistema de classificação de pacientes: construção e validação de um instrumento [dissertação]. São Paulo (SP): Universidade de São Paulo, Escola de Enfermagem; 1996.

16. Fugulin FMT. Sistema de classificação de pacientes: análise das horas de assistência de enfermagem [dissertação]. São Paulo (SP): Universidade de São Paulo, Escola de Enfermagem; 1998.

17. Nonino EAPM, Anselmi ML, Dalmas JC. Avaliação da qualidade do procedimento curativo em pacientes internados em um hospital universitário. Rev Latino-am. Enfermagem [online]. $2008 \mathrm{Fev}$ [acesso 2011 Abr 05]; 16(1): Disponível em: http://www. scielo.br/scielo.php?script=sci_arttext\&pid=S0104$11692008000100010 \& \operatorname{lng}=$ en\&nrm=iso\&tlng=pt.

18. Sala A, coordenador. Parâmetros para o planejamento e dimensionamento da força de trabalho em hospitais gerais. São Paulo (SP): Secretaria de Estado de São Paulo; 2006. p.1-88.

19. Organização Pan-Americana da Saúde. Gestão de redes na OPAS/OMS Brasil: conceitos, práticas e lições aprendidas. Brasília (DF) OPAS/OMA; 2008. p.1-101.

20. Chenso MZB, Haddad MCL, Sêcco IAO, Dorigão MA; Nishiyama MN. Cálculo de pessoal de enfermagem em hospital universitário do Paraná: uma proposta de adequação. Semina Ciênc Biol Saúde. 2004 Jan-Dez; 25(1):81-92.

21. Svaldi JSD, Lunardi Filho WD, Gomes GC. Apropriação e uso de conhecimentos de gestão para a mudança de cultura na enfermagem como disciplina. Texto Contexto Enferm [online]. 2006 Set [acesso 2011 Abr 03]; 15(3): Disponível em: http:/ / 
www.scielo.br/scielo.php?script=sci_arttext\&pid $=$ S0104-07072006000300016.

22. Universidade Estadual de Londrina. Hospital Universitário Regional do Norte do Paraná. Assessoria técnica: relatório anual da Diretoria de Enfermagem. Londrina (PR): UEL; 2009.

23. Silva LG, Haddad MCL, Domansky RC, Vituri DW. Capacidade para o trabalho entre trabalhadores de higiene e limpeza de um hospital universitário. Rev
Eletr Enf [online]. 2010 [acesso 2011 Abr 02]; 12(1): Disponível em: http:/ / www.revistas.ufg.br/index. php/fen/article/viewArticle/5788.

24. Tuomi K, Ilmarinen J, Jahkola A, Katajarinne L, Tulkki A. Índice de capacidade para o trabalho. 2st ed. São Carlos (SP): EdUFSCar; 2005.

25. Nicola AL, Anselmi ML. Dimensionamento de pessoal de enfermagem em um hospital universitário. Rev Bras Enferm. 2005 Mar-Abr; 58(2):186-90. 